\title{
Control and Transport of Passive Particles Using Self-Organized Spinning Micro-Disks
}

\author{
Franco N. Piñan Basualdo ${ }^{(0)}$, Gaurav Gardi, Wendong Wang ${ }^{\circledR}$, Sinan O. Demir ${ }^{\circledR}$, Aude Bolopion ${ }^{\circledR}$, \\ Michaël Gauthier ${ }^{\circledR}$, Pierre Lambert ${ }^{\mathbb{D}}$, and Metin Sitti ${ }^{(1)}$
}

\begin{abstract}
Traditional robotic systems have proven to be instrumental in object manipulation tasks for automated manufacturing processes. Object manipulation in such cases typically involves transport, pick-and-place and assembly of objects using automated conveyors and robotic arms. However, the forces at microscopic scales (e.g., surface tension, Van der Waals, electrostatic) can be qualitatively and quantitatively different from those at macroscopic scales. These forces render the release of objects difficult, and
\end{abstract}

Manuscript received September 9, 2021; accepted December 30, 2021. Date of publication; date of current version. This letter was recommended for publication by Associate Editor X. Wu and Editor X. Liu upon evaluation of the reviewers' comments. The work of Gaurav Gardi was supported by the International Max Planck Research School for Intelligent Systems (IMPRS-IS). The work of Wendong Wang was supported by the UM-SJTU JI. The work of Franco N. Piñan Basualdo and Pierre Lambert were supported in part by the Belgian Science Policy Office (IAP 7/38 MicroMAST), and in part by the Fonds de la Recherche Scientifique under Grant PDR T.0129.18. The work of Franco N. Piñan Basualdo, Aude Bolopion, and Michaël Gauthier were supported by the French ANR Program Equipex ROBOTEX Project under Grant ANR-10-EQPX-44-01, in part by the EUR EIPHI Program under Grant ANR-17-EURE-0002, and in part by the French Agence Nationale de la Recherche and the Swiss National Science Foundation through the CoDiCell Project under Grant ANR-17-CE33-0009. The work of Sinan O. Demir was supported by the Ministry of National Education of the Republic of Turkey through the Doctoral Scholarship. This work was supported by the Max Planck Society. (Franco N. Piñan Basualdo and Gaurav Gardi are co-first authors). (corresponding authors: Wendong Wang; Metin Sitti).

Franco N. Piñan Basualdo is with the Physical Intelligence Department, Max Planck Institute for Intelligent Systems, 70569 Stuttgart, Germany, with the FEMTO-ST Institute, CNRS, Université Bourgogne Franche-Comté, F25000 Besançon, France, and also with the Transfers Interfaces and Processes (TIPs), Université Libre de Bruxelles, 1050 Brussels, Belgium (e-mail: Franco.Pinan.Basualdo@ulb.be).

Gaurav Gardi is with the Physical Intelligence Department, Max Planck Institute for Intelligent Systems, 70569 Stuttgart, Germany (e-mail: gaurav.gardi2@gmail.com).

Wendong Wang is with the University of Michigan - Shanghai Jiao Tong University Joint Institute, Shanghai 200240, China (e-mail: wendong.wang@sjtu.edu.cn).

Sinan O. Demir is with the Physical Intelligence Department, Max Planck Institute for Intelligent Systems, 70569 Stuttgart, Germany, and also with the Stuttgart Center for Simulation Science (SC SimTech), University of Stuttgart, 70569 Stuttgart, Germany (e-mail: sozgundemir@gmail.com).

Aude Bolopion and Michaël Gauthier are with the FEMTO-ST Institute, CNRS, Université Bourgogne Franche-Comté, F-25000 Besançon, France (email: aude.bolopion@femto-st.fr; michael.gauthier@femto-st.fr).

Pierre Lambert is with the Transfers Interfaces and Processes (TIPs), Université Libre de Bruxelles, 1050 Brussels, Belgium (e-mail: pierre.lambert@ulb.ac.be).

Metin Sitti is with the Physical Intelligence Department, Max Planck Institute for Intelligent Systems, 70569 Stuttgart, Germany, with the School of Medicine and College of Engineering, Koç University, Istanbul 34450, Turkey, and also with the the Institute for Biomedical Engineering, ETH Zurich, 8092 Zurich, Switzerland (e-mail: sitti@is.mpg.de).

This letter has supplementary downloadable material available at https://doi.org/10.1109/LRA.2022.3143306, provided by the authors.

Digital Object Identifier 10.1109/LRA.2022.3143306 hence, traditional systems cannot be directly transferred to small scales (below a few millimeters). Consequently, novel micro-robotic manipulation systems have to be designed to take into account these scaling effects. Such systems could be beneficial for microfabrication processes and for biological studies. Here, we show autonomous position control of passive particles floating at the air-water interface using a collective of self-organized spinning micro-disks with a diameter of $300 \mu \mathrm{m}$. First, we show that the spinning micro-disks collectives generate azimuthal flows that cause passive particles to orbit around them. We then develop a closed-loop controller to demonstrate autonomous position control of passive particles without physical contact. Finally, we showcase the capability of our system to split from an expanded to several circular collectives while holding the particle at a fixed target. Our system's contact-free object manipulation capability could be used for transporting delicate biological objects and for guiding self-assembly of passive objects for micro-fabrication.

Index Terms-Automation at micro-nano scales, micro/nano robots, swarm robotics.

\section{INTRODUCTION}

$\mathbf{R}$ OBOT-ASSISTED assembly lines have revolutionized the manufacturing industry. Specially, these systems are instrumental in the automation of object manipulation, transport and assembly tasks. Typical robotic systems in industries incorporate the conveyor system for automated transport of objects and are integrated with robotic arms for pick-and-place and assembly of objects. However, at millimeter scale and below, robotic systems need to overcome the barrier presented by the dominant physical interactions at such scales, like surface tension, Van der Waals interactions, and electrostatic attraction [1], [2]. Considering these effects, several systems have been proposed for manipulation at the micro-scale. Most of these systems operate based on physical contact with the passive object [3]-[9] while a few systems make use of the fluid environment around the objects for non-contact manipulation [10]-[16].

Contact-based object manipulation is normally achieved by controlling an external stimulus (for example, magnetic field) in the environment to move one or more micro-robots that, in turn, push or pull the passive objects. However, contact-based pushing and pulling is less preferable due to the adhesion between the manipulated object and the manipulator, impeding the release of the former. Non-contact manipulation can be performed via the generation of flows within the fluid, which in turn move the object. These flows could be created either using fixed platforms [12], [13] or by mobile micro-objects [14]-[17]. The fixed platforms can be made either using active components 


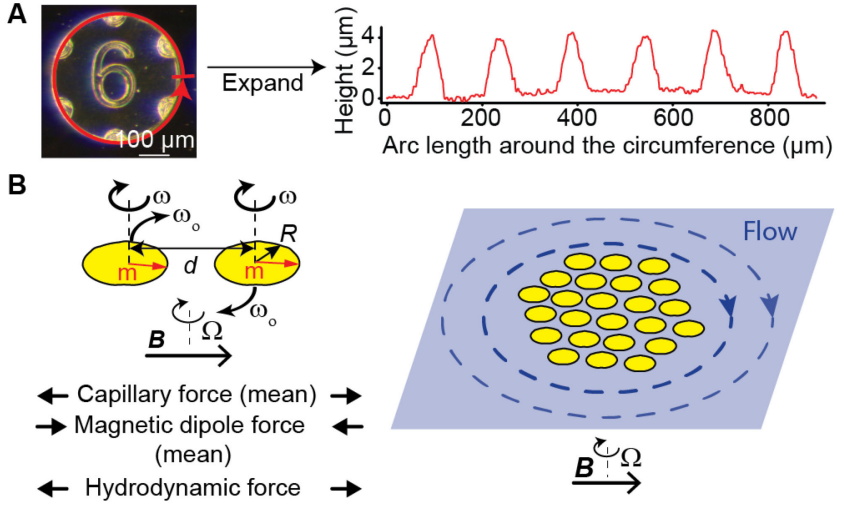

Fig. 1. Behavior of spinning micro-disks. (A) Optical microscope image of a single micro-disk (left) and the edge height profile (right). Each micro-disk has 6 bumps along its edge which can been seen in the edge-height profile (right). The number 6 on the micro-disk is to identify the orientation of the disk. (B) Schematic of a pair of micro-disks spinning on air-water interface (left) and a collective with generated flow around it (right). The torque due to the external magnetic field (spin speed $\Omega$ ), rotates each disk (spin speed $\omega$ ). The disks interact with each other via three pairwise interactions: capillary, magnetic dipole-dipole and the hydrodynamic lift. The disks also generate an azimuthal flow on the surface of water when they spin, and they orbit around each other at orbiting speed $\omega_{0}$, due to this flow field.

like photo-chemically active Janus pillars [12] or using acoustic transducers [13]. Micro-robotic systems that have been developed for object manipulation either use a single robot [15]-[17] or a swarm [14]. However, all of these systems either cannot dynamically reconfigure the flows in real-time or they can only manipulate objects near a solid substrate, where the objects need to overcome the surface friction.

The systems mentioned earlier cannot be used for some applications that require manipulation at fluid-fluid interface. Biological studies on the behavior of biological materials such as protein [18]-[20], epithelial cells [21]-[23] and other microorganisms [24], [25] at the air-water interface are of both fundamental and practical importance. These studies could benefit from robotic systems that autonomously manipulate floating objects at the air-water interface.

Moreover, these micro-scale robotic systems could be useful for guiding capillary self-assembly [26] of simple objects to form complex shapes, which can be useful for nanoscale packaging [27]. One such system has been developed recently [11] which uses laser-induced thermocapillary flow to manipulate a few objects in parallel. However, it requires an extremely clean air-water interface for it to work, which limits the working time of the system to a few minutes. Another possibility is to use a swarm of micro-robots that can generate flows on a fluid surface. It has been shown that a collective of magnetic micro-disks spinning at the air-water interface self-organize into groups and orbit around each other [28]-[30]. These collectives generate azimuthal flows around themselves that could be used for transporting an object [10]. Variation of the rotation speed of the external magnetic field changes the patterns of spinning micro-disks, which in turn reconfigure the flow at the air-water interface. This ability to reconfigure the flow makes the system suitable for non-contact object manipulation at the air-water interface.

In this study, we demonstrate feedback control of passive objects at the air-water interface using a collective of spinning magnetic micro-disks. We use a few soft-magnetic rods to concentrate the magnetic flux in some areas, thus creating magnetic traps. First, we demonstrate that by tuning the spinning speed of the external magnetic field, we can localize the disks within the magnetic trap, forming several circular collectives, or allow them to escape to neighboring traps, thereby forming a single rod-like collective. Next, we show the transport of passive particles around the collective and how the transport speed can be controlled by periodically switching the magnetic rotation direction. Utilizing the above-mentioned results, we then demonstrate the autonomous transport of $\approx 1^{\sim} \mathrm{mm}$ sized objects at the air-water interface. We implement a real-time closed-loop controller to autonomously control the position of passive particles. Our system has the potential to be used for sorting biological cells and to guide self-assembly of passive objects into non-trivial configurations for micro/milli-scale assembly towards the fabrication of complex objects.

\section{BEHAVIOR OF THE SPINNING MicRO-DISKS}

The experimental setup consists of two pairs of Helmholtz coils to generate a uniform rotating magnetic field along the horizontal plane. Inside the work space of the coils, we place an arena with water on top of which magnetic micro-disks float. The disks are corrugated along the edges [29] (Fig. 1a) and are sputtered with cobalt to generate a magnetic dipole (see Appendix for details on fabrication). These corrugations deform the air-water interface, thereby inducing orientation dependent capillary interactions. The magnetic dipole of the disks causes an orientation dependent dipole-dipole interaction between the disks. During normal operation, the magnetic field magnitude is kept constant and it rotates with an imposed angular velocity $\Omega$, this is

$$
\mathbf{B}=B_{0} \cos (\Omega t) \cdot \hat{x}+B_{0} \sin (\Omega t) \cdot \hat{y}
$$

where we fixed $B_{0}=7 \mathrm{mT}$. The magnetic disks floating at the interface experience a torque from the externally imposed magnetic field and begin to rotate with an angular velocity $\omega$. As long as the magnetic rotation speed $\Omega$ is not too high $(\Omega<$ $\Omega_{\text {step-out }}$, the disks will rotate with the same average angular speed $(\bar{\omega}=\Omega)$. When two rotating disks are close to each other, they interact in three different ways: capillary, magnetic and hydrodynamic interaction [28]. The capillary interaction can be repulsive or attractive depending on the relative orientation of the disks, but the average interaction along one turn is repulsive. The magnetic interaction can also be attractive or repulsive depending on the relative orientation of the disks' magnetic dipoles, but in this case the average interaction is attractive. Finally, there is the hydrodynamic interaction, that is always repulsive and it depends on the spinning speed of the disks $(\omega)$. The disks also generate an azimuthal flow when they rotate, making them orbit around a common center of mass [29]. All these interactions combined make the disks stay at a stable distance from each other 
B
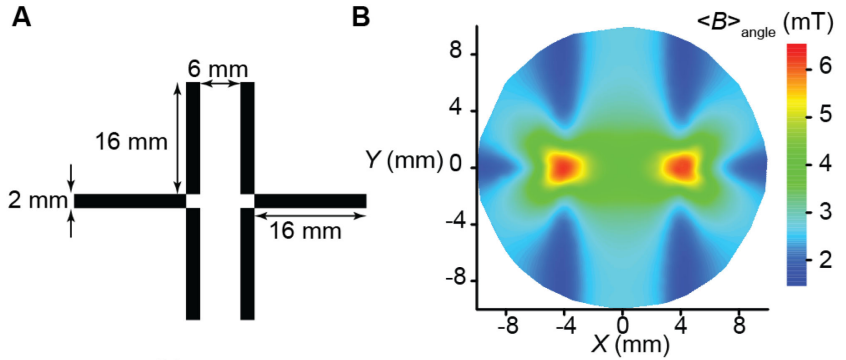

C
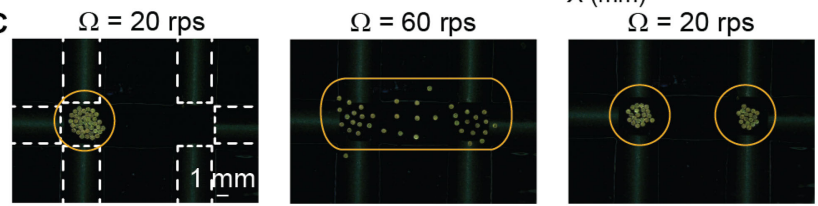

Fig. 2. Spinning micro-disks in magnetic traps. (A) Schematic of the arrangement of soft magnetic ferrite rods that create a double magnetic trap when the external magnetic field is switched on. The ferrite rods concentrate the magnetic flux near its extremes. (B) COMSOL simulation showing the angle-average magnetic flux density corresponding to the arrangement in $(\mathrm{A})$ placed in uniform rotating magnetic field $(10 \mathrm{mT})$. The arrangement results in a double magnetic trap with minima at the center of each square gap created by the rods. (C) Representative images showing the behavior of disks in the double magnetic trap. At lower spinning speed ( $\Omega=20 \mathrm{rps}$ ) the disks were localized to the trap to the left (left) and at higher spin speed ( $\Omega=60 \mathrm{rps}$ ) they expanded and occupy the neighboring trap (center). Switching from $\Omega=60 \mathrm{rps}$ to $\Omega=20 \mathrm{rps}$, the long collective can be split into two small circular collectives, localized in the two traps (right). The white boxes in (C) outline the ferrite rods.
(Fig. 1B) while orbiting. These interactions can be extended to more disks, that form a collective with a characteristic distance between particles depending on the rotation speed $\Omega$ [30].

The system of spinning disks described above can be enriched by introducing a magnetic trap, an area with stronger magnetic field that attracts the disks. Such traps can be created with ferrite bars that concentrate the magnetic field at their extremes, attracting the disks there. Moreover, two traps can be created next to each other arranging the bars as shown in Fig. 2A. The resulting magnetic trap can be seen in Fig. 2B. The strength of the trap can be controlled by changing the magnitude of the external magnetic field. Under this configuration, the behavior of the collectives can radically change depending on $\Omega$ as shown in Fig. 2C. When the rotation speed is below a given threshold, the disks in one trap cannot escape to the other one. Above this threshold, because of the increased hydrodynamic repulsion between the disks, the disks can escape from one trap to the other, filling the space in between and forming an elongated collective. If now $\Omega$ is decreased again, the collective breaks into two localized groups.

\section{RESULTS}

\section{A. Manipulation of Passive Particles}

In the previously described setup, the rotation of each magnetic disk generates an azimuthal flow around it that superimposes into a flow around the entire collective [28]. This flow can be used to propel passive particles as shown in Fig. 3A and $\mathrm{B}$ for $\Omega=20 \mathrm{rps}$ and $\Omega=60 \mathrm{rps}$ respectively. In order to accurately control the particle, we need to control the intensity of
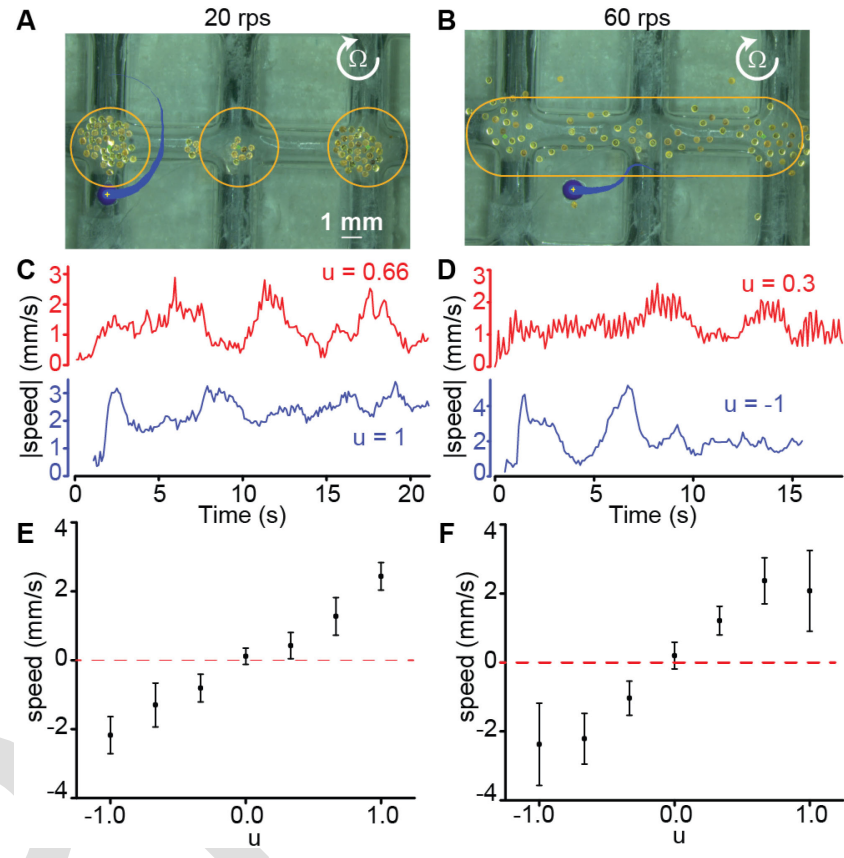

Fig. 3. Passive particle transport by the flow generated by the collective. Image showing the passive particle orbiting around the collective at (A) $20 \mathrm{rps}$ and (B) 60 rps due to the flow generated by the disks. By spinning the disks clockwise for a time $t_{\mathrm{CW}}=\Delta t(u+1) / 2$, where $u \in[-1,1]$ is the control variable, and anti-clockwise for a time $\Delta t-t_{\mathrm{CW}}$ at same $\Omega$, the average speed of the passive particle can be tuned. Plot of speed vs time of a $1 \mathrm{~mm}$ polystyrene bead for different values of $u$ at (C) $20 \mathrm{rps}$, and (D) $60 \mathrm{rps}$. Plot of average speed of a $1 \mathrm{~mm}$ polystyrene bead at (E) $20 \mathrm{rps}$, and (F) $60 \mathrm{rps}$ for different values of $u$. The standard deviations in $(\mathrm{E})$ and $(\mathrm{F})$ were calculated from trajectories, 10 to $30 \mathrm{~s}$ long. Blue line in (A) and (B) represent trajectory of the particle over the last $5 \mathrm{~s}$.

the generated flow. The simplest way to control the flow intensity is to adjust the magnetic rotation speed $\Omega$. However, modifying $\Omega$ also affects the interactions between the disks and, therefore, the behavior of the collective as explained in the previous section.

One way to adjust the flow intensity without strongly affecting the collective is to keep the absolute rotation speed $\Omega$ constant, but periodically change its direction. In other words, the magnetic field, and thus the disks, are rotated for a time $t_{\mathrm{CW}}$ in the clockwise direction and for a time $t_{\mathrm{CCW}}=\Delta t-t_{\mathrm{CW}}$ in the anti-clockwise direction. We can then define the first duration as

$$
t_{\mathrm{CW}}=\Delta t \frac{u+1}{2}
$$

where $u \in[-1,1]$ is the control variable. One limitation of the current setup is that a waveform cannot be interrupted midway, thus limiting $t_{\mathrm{CW}}$ and $\Delta t$ to whole periods. Consequently, a low value of $\Delta t$ would leave only a few possible values for $t_{\mathrm{CW}}$, and a too large $\Delta t$ would make the passive particle oscillate back and forth. We found a good compromise for $\Delta t=300 \mathrm{~ms}$, leaving 7 possible values for $t_{\mathrm{CW}}$ when $\Omega=20 \mathrm{rps}$ (split configuration), and 19 when $\Omega=60 \mathrm{rps}$ (expanded configuration). The response of the system under a sudden change of $u$ from zero to a given value is shown in Fig. 3C and D. Two observations can be made from these results. First, the inertia of the system is negligible, with the particle accelerating almost immediately. 


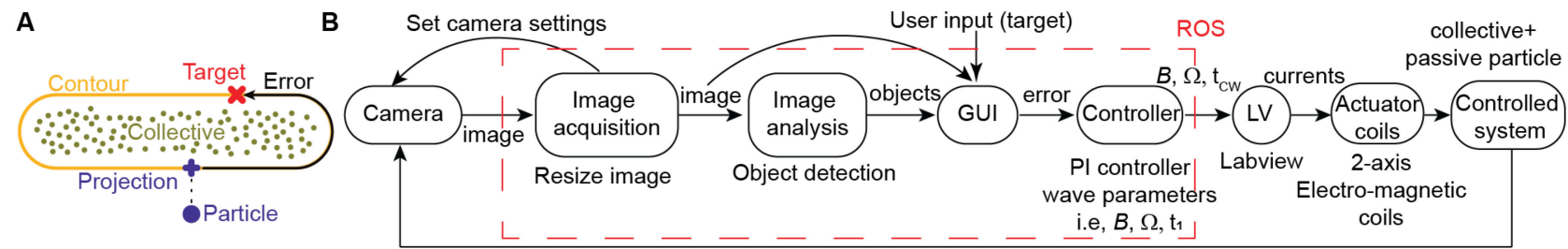

Fig. 4. Schematic of the error calculation and the control architecture. (A) Error calculation. The particle position is projected on the collective's contour. The error is then calculated as the distance to the target along the contour. (B) Control architecture. Images acquired (image acquisition) using a camera are analyzed (image analysis) to detect the passive particle. The target position is received from the user through a GUI. A PI controller is used to compute the waveform parameters $\left(\Omega\right.$ and $\left.t_{\mathrm{CW}}\right)$. The LabVIEW program computes and commands the currents to the coils accordingly. The coils then generate a magnetic field that drives the collective, which in turn drives the passive particles (controlled system). The control architecture was implemented using a custom-made python package with Robot Operating System (ROS) for communication between different modules.

Second, the instantaneous particle velocity strongly fluctuates. These fluctuations can be explained by the fact that the collective is constantly reorganizing itself, thus affecting the generated flow and passive particle velocity. Nevertheless, the effectiveness of the proposed technique can be seen in Fig. 3E and F, where the average velocity of the particle resulted approximately proportional to $u$. The fact that both values of $\Omega$ produced similar velocities could be explained by considering that even if at lower rotation speeds the individual effect of each disk is weaker, they aggregate together and the particle gets closer to the collective (because of the reduced hydrodynamic interaction), thus compensating the weakening of the individual contributions.

Intuitively, one may think that the presented strategy could be used to stop the flow, and thus the particle, by imposing $u=0$. Although, doing so effectively diminish the average velocity of the particle (Fig. 3E and F), the mean velocity is not exactly zero and the particle continues to advance with an average speed of around $0.1 \mathrm{~mm} / \mathrm{s}$ and $0.2 \mathrm{~mm} / \mathrm{s}$ for $\Omega=20 \mathrm{rps}$ and $\Omega=60 \mathrm{rps}$ respectively. Moreover, the passive particle velocity fluctuations, make it impossible to predict in advance the necessary input to obtain a given displacement, thus making necessary the implementation of a closed-loop controller.

\section{B. Closed-Loop Position Control of Passive Particles}

Although the previous results were encouraging, they also evidenced the need for a closed-loop control. This is, the value of $u$ should be updated as a function of the particle position relative to an imposed target. Concretely, we need to identify the passive particle position, compute the error to a target, and update the value of $u$ accordingly. Since the particle can only be controlled around the collective, both the particle position and the target are projected on a contour - composed of two straight lines and two semicircles that approximate the trajectory traversed by the passive particle - and the error is computed along it (Fig. 4A).

The implementation of the controller is split into two computers communicating through TCP/IP protocol, one executing several Python scripts with Robot Operating System (ROS) for communication between them, and one running LabVIEW. A schematic of the closed-loop system is shown in Fig. 4B. The ROS computer is in charge of image acquisition, image analysis, user interface and controller calculations. The image acquisition subsystem sets the camera and launches the image

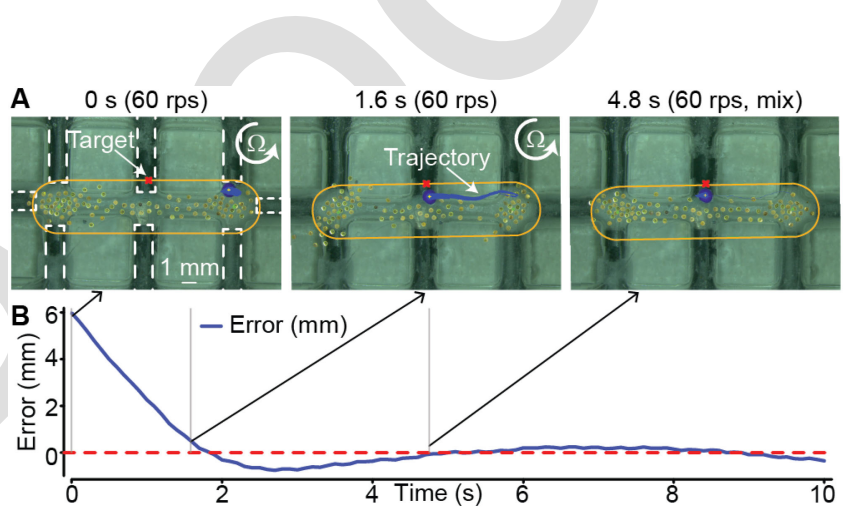

Fig. 5. Autonomous control of passive particles using expanded collective. (A) Image sequence showing the autonomous position control of a $1 \mathrm{~mm}$ sphere while the collective is in expanded state at $\Omega=60 \mathrm{rps}$. (B) Plot of error vs time corresponding to the experiment in (B). Blue lines in (A) represent trajectory of the particle over the last $5 \mathrm{~s}$.

acquisition. The image analysis, transforms the image to HSV representation, and filters the $\mathrm{H}$ channel to detect blue objects. The user interface (GUI) displays the image to the user, receives user inputs (target and mode), and calculates the error $(e)$. The control subsystem implements a PI controller to compute $u$ as

$$
u(t)=K_{p}\left(e(t)+\frac{1}{T_{i}} \int_{0}^{t} e(\tau) d \tau\right),
$$

where $e$ is the error, $K_{p}$ is the proportional gain and $T_{i}$ is the integral time. Then, the control variable was saturated to $|u| \leq$ 0.9 , in order to reduce the collective deformations and to stay in the linear regime (see Fig. 3E and F). The control subsystem also computes $t_{\mathrm{CW}}$ using (2). The LabVIEW computer receives the waveform parameters ( $\Omega$ and $t_{\mathrm{CW}}$ ) from the ROS side, and computes the signal for each coil driver.

In the expanded configuration ( $\Omega=60 \mathrm{rps}$ ), the particle can be controlled all around the collective. We implemented the previously described controller with a proportional gain $K_{p}=$ $0.22 \mathrm{~mm}^{-1}$ and an integral time $T_{i}=200 \mathrm{~s}$, and the results are shown in Fig. 5A and B (also see accompanying video). This controller successfully drives the particle and stabilizes it around the target with a precision of approximately $0.5 \mathrm{~mm}$. Although a derivative action could improve the transient response, the previously discussed velocity fluctuations make it not trivial to implement without introducing fluctuations in the control variable $u$. The same controller was used in the split configuration 

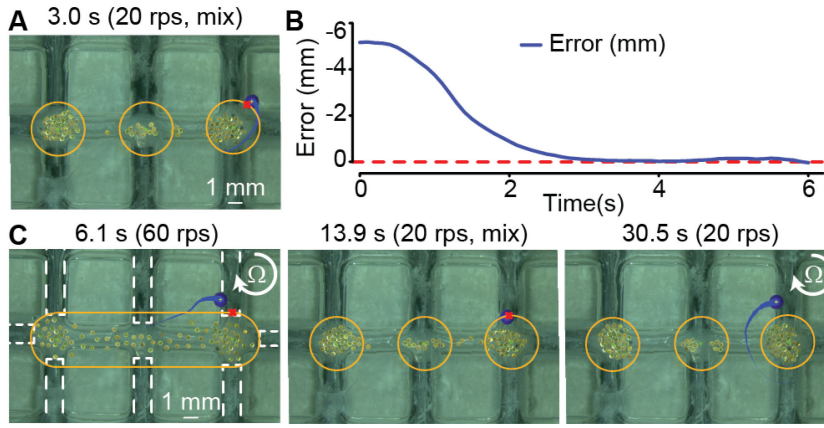
$13.9 \mathrm{~s}(20 \mathrm{rps}, \mathrm{mix})$
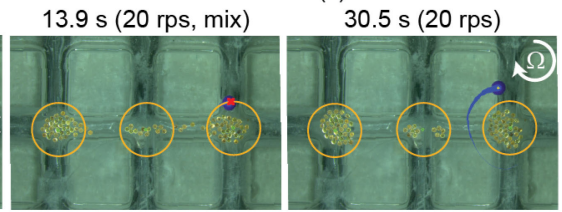

Fig. 6. Autonomous control of passive particles using splitting behavior. (A) Image showing the position control of a $1 \mathrm{~mm}$ sphere while the collective is split at $\Omega=20$ rps. (B) Plot of error vs time corresponding to the experiment in (A). (C) Image sequence showing the transition from expanded collective at $\Omega=60 \mathrm{rps}$, to small circular collectives at $\Omega=20 \mathrm{rps}$, while holding the particle at the target position. The particle was first moved to a target position while the collective was expanded, then it split to circular collectives while still holding the particle at the target. Finally, the particle was allowed to orbit around the circular collective to the right. Blue lines (A) and (C) represent trajectory of the particle over last $5 \mathrm{~s}$.
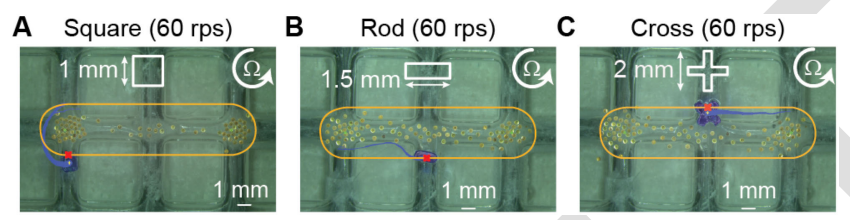

Fig. 7. Control of passive particles of different shapes. The images showing the trajectory of (A) $1 \mathrm{~mm}$ square, (B) $1.5 \mathrm{~mm}$ rod, and (C) $2 \mathrm{~mm}$ cross shaped passive particle (3D printed) while the collective is in expanded state at $\Omega=$ $60 \mathrm{rps}$. The blue lines represent the trajectory of the passive particle over the last $5 \mathrm{~s}$. The system successfully controlled the particles without adjusting the controller gains.

( $\Omega=20 \mathrm{rps}$ ) to control the particle around one of the small collectives, and the results are shown in Fig. 6A and B. In this case, the particle is stabilized around the target with a precision of approximately $0.3 \mathrm{~mm}$.

The main advantage of collective systems over single active particles is that collective systems can reconfigure on demand. In particular, our system allows us to split the collective while holding the particle in place to later control the particle around one of the smaller collectives as shown in Fig. 6C (also see accompanying video). In this example, the particle is first driven to one of the extremes of the expanded collective, stabilized there while the collective was split, and finally the control is set to $u=$ 1 to make the particle orbit around the smaller collective. This can be reversed by first stopping the particle, then expanding, and finally controlling the particle around the expanded collective.

Another advantage of this system is that, since it is driven by the fluid motion, the shape and material of the passive particle do not have a significant impact on its response. Therefore, passive particles with different shapes can be controlled without having to modify the controller gains, as shown in Fig. 7.

\section{CONCLUSION}

In summary, we presented a novel non-contact manipulation platform that makes use of the flow created by a collective of rotating magnetic micro-disks to control a passive particle around it. First, we showed how the collective exhibits two distinct behaviors depending on the rotation speed $\Omega$. Namely, the system expands along several magnetic traps for fast rotation, and splits into several small collectives for slow rotations. Then we showed that we can decouple the collective behavior from its effect on the passive particle by keeping the magnetic field magnitude $B_{0}$ and instantaneous rotation speed $\Omega$ constant, but periodically switching the rotation direction. While this allowed us to tune the passive particle's velocity, the observed velocity fluctuations and residual velocity for zero input evidenced the need for a feedback controller. We implemented such a controller by identifying the passive particle's position through image analysis, and updating the control parameters accordingly. This controller successfully drove the particle to the target position and stabilized it there. Moreover, taking advantage of the collective's behaviors, we can split the collective and trap the passive particle in an orbit around one of the small collectives.

The main limitation of the system in its current state is that passive particles can only be controlled along the contour of the collective, and the position of the particle perpendicular to this contour depends on its interaction with the collective. Another drawback is that all the passive particles around the collective experience the same actuation (clockwise or anti-clockwise). This renders the independent control of several particles difficult. One workaround could be to separate the particles by using the splitting capability of the collective, thereby differentiating the effect of the flows on each particle. Finally, the fluctuations on the collective structure (magnetic particles positions) may affect the propulsion. For example, in the expanded configuration the particle can occasionally break through the collective and orbit around one of the external traps, thus preventing the collective from reforming. More advanced controllers could consider the collective structure to predict such fluctuations and act accordingly. For example, prevent the particle form breaking through the collective or, if it does, reduce its speed to allow the collective to reconfigure. Moreover, it has been proven that iterative learning algorithms can improve the performance of non-contact manipulation [17] for single actuators in the fluid bulk, which could be extended to collectives at the fluid interface.

The developed system has several potential applications in micromanipulation. First, it could be used as a micro conveyor belt to displace particles or assemblies in a micro-fabrication device. A scaled down version of the system could be used to transport delicate biological material, because of the non-contact manipulation capability of the system. Moreover, the splitting capability can be exploited to manipulate multiple particles simultaneously and, possibly, assemble them. Another capability of the system could be the sorting of particles, with possible application within a lab-on-a-chip device.

\section{APPENDIX}

Fabrication of the micro-disks: A CAD model for the microdisks was created using Grasshopper plugin in Rhinoceros 3D software. The capillary bumps are cosine profiles with amplitude $2 \mu \mathrm{m}$, spread over an arc angle of $30^{\circ}$. The micro-disks were then $3 \mathrm{D}$ printed using two photon lithography (Nanoscribe) and 
a $500 \mathrm{~nm}$ thick cobalt layer was sputtered (using Kurt J. Lesker NANO 36) on them to generate an in-plane magnetic dipole. Finally, a $60 \mathrm{~nm}$ thick gold layer was sputtered on top to protect the cobalt from oxidizing. The optical microscope images were taken on Zeiss Discovery V12 using Basler camera acA1300200uc.

Design of the soft magnetic traps: Soft magnetic traps were designed using $2 \mathrm{~mm}$ ferrite rods. These rods exhibit soft magnetic behavior, that is, they get magnetized inside an external magnetic field and they lose magnetization almost entirely when the external magnetic field is switched off. The distance between the magnetic traps was empirically adjusted. This distance influences the magnitude of the magnetic barrier that prevents the disks from escaping from a trap. We found that at $6 \mathrm{~mm}$ separation between the two magnetic traps, we could obtain the two distinct behaviors (localized at $20 \mathrm{rps}$ and expanded at $60 \mathrm{rps}$ ) of the collective and transition between them.

Arena for the experiments: The experiments were performed inside a rectangular arena that was 3D printed using stereolithography (SLA) printers (Formlabs Form 3). The arena was designed to accommodate the ferrite rods.

Passive particle fabrication: Passive particles (square, rod and cross) were 3D printed using Stereolithography (SLA) printers (Formlabs) and clear V4 resin.

\section{REFERENCES}

[1] M. Sitti, Mobile microrobotics. Cambridge, MA: The MIT Press, 2017. [Online]. Available: https://mitpress.mit.edu/books/mobilemicrorobotics

[2] R. S. Fearing, "Survey of sticking effects for micro parts handling," in Proc. IEEE/RSJ Int. Conf. Intell. Robots Syst. Hum. Robot Interaction Cooperative Robots, 1995, pp. 212-217.

[3] X. Dong and M. Sitti, "Controlling two-dimensional collective formation and cooperative behavior of magnetic microrobot swarms," Int. J. Robot. Res., vol. 39, no. 5, pp. 617-638, 2020.

[4] W. Wang, V. Kishore, L. Koens, E. Lauga, and M. Sitti, "Collectives of spinning mobile microrobots for navigation and object manipulation at the air-water interface," in Proc. IEEE/RSJ Int. Conf. Intell. Robots Syst., 2018, pp. 1-9.

[5] X.Fan, X. Dong, A. C. Karacakol, H. Xie, and M. Sitti, "Reconfigurable multifunctional ferrofluid droplet robots," Proc. Nat. Acad. Sci., vol. 117, no. 45, pp. 27916-27926, 2020. [Online]. Available: https://www.pnas. org/content/117/45/27916

[6] B. V. Johnson, N. Esantsi, and D. J. Cappelleri, "Design of the $\mu$ MAZE platform and microrobots for independent control and micromanipulation tasks," IEEE Robot. Automat. Lett., vol. 5, no. 4, pp.5677-5684, Oct. 2020.

[7] M. A. Rahman, J. Cheng, Z. Wang, and A. T. Ohta, "Cooperative micromanipulation using the independent actuation of fifty microrobots in parallel," Sci. Rep., vol. 7, no. 1, pp. 1-11, 2017.

[8] G. Grosjean, M. Hubert, and N. Vandewalle, "Magnetocapillary selfassemblies: Locomotion and micromanipulation along a liquid interface," Adv. Colloid Interface Sci., vol. 255, pp. 84-93, 2018.
[9] A. Barbot, H. Tan, M. Power, F. Seichepine, and G.-Z. Yang, "Floating magnetic microrobots for fiber functionalization," Sci. Robot., vol. 4, no. $34,2019$.

[10] B. A. Grzybowski, M. Radkowski, C. J. Campbell, J. N. Lee, and G. M. Whitesides, "Self-assembling fluidic machines," Appl. Phys. Lett., vol. 84, no. 10, pp. 1798-1800, 2004.

[11] F. N. P. Basualdo, A. Bolopion, M. Gauthier, and P. Lambert, "A microrobotic platform actuated by thermocapillary flows for manipulation at the air-water interface," Sci. Robot., vol. 6, no. 52, 2021, Art. no. eabd3557.

[12] T. Yu et al., "Microchannels with self-pumping walls," ACS Nano, vol. 14, no. 10, pp. 13673-13680, 2020.

[13] K. Melde, A. G. Mark, T. Qiu, and P. Fischer, "Holograms for acoustics," Nature, vol. 537, no. 7621, pp. 518-522, 2016.

[14] H. Xie et al., "Reconfigurable magnetic microrobot swarm: Multimode transformation, locomotion, and manipulation," Sci. Robot., vol. 4, no. 28, 2019.

[15] Z. Ye and M. Sitti, "Dynamic trapping and two-dimensional transport of swimming microorganisms using a rotating magnetic microrobot," Lab Chip, vol. 14, no. 13, pp. 2177-2182, 2014.

[16] Z. Ye, E. Diller, and M. Sitti, "Micro-manipulation using rotational fluid flows induced by remote magnetic micro-manipulators," J. Appl. Phys., vol. 112, no. 6, 2012, Art. no. 064912

[17] C. Pawashe, S. Floyd, E. Diller, and M. Sitti, "Two-dimensional autonomous microparticle manipulation strategies for magnetic microrobots in fluidic environments," IEEE Trans. Robot., vol. 28, no. 2, pp. 467-477, Apr. 2012.

[18] P.-C. Chiang, K. Tanady, L.-T. Huang, and L. Chao, "Rupturing giant plasma membrane vesicles to form micron-sized supported cell plasma membranes with native transmembrane proteins," Sci. Rep., vol. 7, no. 1, pp. 1-8, 2017.

[19] Y.-L. Huang, "Experiments and modeling on an air-water interface populated by biological molecules," Ph.D. dissertation, Université JosephFourier-Grenoble I., 2009.

[20] M. J. Gálvez-Ruiz, "Different approaches to study protein films at air/water interface," Adv. Colloid Interface Sci., vol. 247, pp. 533-542, 2017.

[21] R. Tchao, "Epithelial cell interaction in air-liquid interface culture," In Vitro Cellular Devlop. Biol., vol. 25, no. 5, pp. 460-465, 1989.

[22] T. Bluhmki et al., "Development of a miniaturized 96-transwell air-liquid interface human small airway epithelial model," Sci. Rep., vol. 10, no. 1, pp. $1-14,2020$

[23] H. Wang et al., "Establishment and comparison of air-liquid interface culture systems for primary and immortalized swine tracheal epithelial cells," BMC Cell Biol., vol. 19, no. 1, pp. 1-10, 2018.

[24] M. Morse, A. Huang, G. Li, M. R. Maxey, and J. X. Tang, "Molecular adsorption steers bacterial swimming at the air/water interface," Biophysical J., vol. 105, no. 1, pp. 21-28, 2013.

[25] J. Ferracci, H. Ueno, K. Numayama-Tsuruta, Y. Imai, T. Yamaguchi, and T. Ishikawa, "Entrapment of ciliates at the water-air interface," PLoS One, vol. 8, no. 10, 2013, Art. no. 75238 .

[26] D. Vella and L. Mahadevan, "The "cheerios effect", Amer. J. Phys., vol. 73, no. 9, pp. 817-825, 2005

[27] C. Morris, S. Stauth, and B. Parviz, "Self-assembly for microscale and nanoscale packaging: Steps toward self-packaging," IEEE Trans. Adv. Packag., vol. 28, no. 4, pp. 600-611, Nov. 2005.

[28] B. A. Grzybowski, H. A. Stone, and G. M. Whitesides, "Dynamics of self assembly of magnetized disks rotating at the liquid-air interface," Proc. Nat. Acad. Sci., vol. 99, no. 7, pp. 4147-4151, 2002.

[29] W. Wang, J. Giltinan, S. Zakharchenko, and M. Sitti, "Dynamic and programmable self-assembly of micro-rafts at the air-water interface," $S c i$. Adv., vol. 3, no. 5, 2017, Art. no. e1602522.

[30] W. Wang et al., "Order and information in the phases of a torque-driven collective system," 2019, arXiv:1910.11226. 